\title{
Quantification of left atrial volume using cardiac magnetic resonance imaging: comparison of left atrial volume index measurements using the Simpson's and bi-plane area-length methods
}

Hareeprasad R Vongooru, Ashenafi M Tamene*, Prabhjot S Nijjar, Sue Duval, Uma Valeti

From 17th Annual SCMR Scientific Sessions

New Orleans, LA, USA. 16-19 January 2014

\section{Background}

Increased left atrial volume index (LAVI) has been shown to be an independent predictor of hard cardiovascular events. It can be determined by calculating left atrial (LA) volume through the use of either Simpson's method or area-length method and indexing it to body surface area (BSA). Although there is no standardized method of calculating LA volume using Cardiac Magnetic Resonance Imaging (CMR), the aforementioned methods, which were originally validated for 2-dimensional echocardiography, have been used. We sought to assess the agreement between LAVI, obtained with the use of cardiac gated steady state free precession cine pulse sequence of contiguous short axis slices $\left(\mathrm{LAVI}_{1}\right)$ and area-length method $\left(\mathrm{LAVI}_{2}\right)$.

\section{Methods}

Thirty-six patients (28 males, age $47.6 \pm 16.7$ years) who had adequate CMR imaging to analyze LA volumes using the two methods were retrospectively identified. LAVI was calculated using Simpson's stack of discs method on short axis cine images (acquired as $8 \mathrm{~mm}$ slices with inter slice gap of $2 \mathrm{~mm}$ or $6 \mathrm{~mm}$ slices with no gap). $\mathrm{LAVI}_{2}$ was measured using the American Society of Echocardiography guidelines. Qmass software (Medis) was used to trace LA areas and volumes.

\section{Results}

The mean $( \pm \mathrm{SD})$ values for $\mathrm{LAVI}_{1}$ and $\mathrm{LAVI}_{2}$ were $44.9( \pm 17.3)$ and $45.6( \pm 18.4)$ respectively. There was a significant correlation between $\mathrm{LAVI}_{1}$ and $\mathrm{LAVI}_{2}$
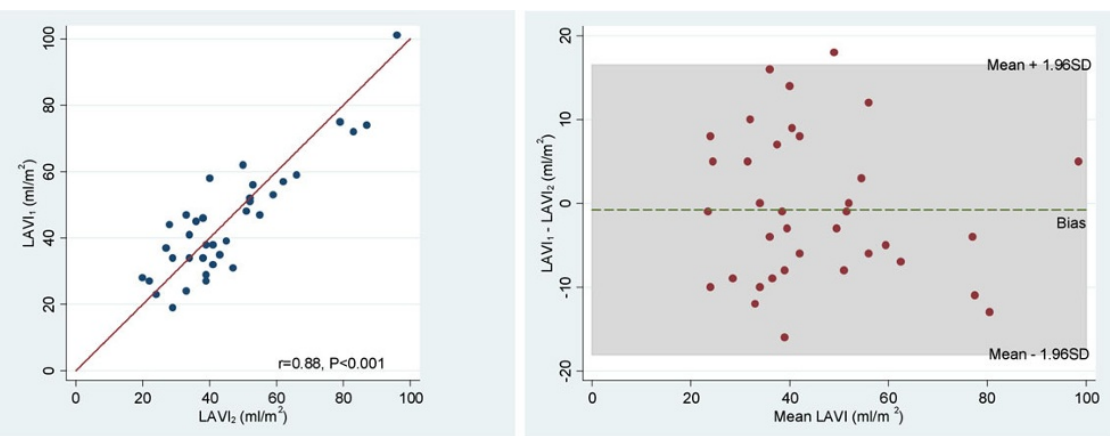

Figure 1 A: Correlation plot of LAVI1 and LAVI2 Figure 1B: Bland-Altman plot of LAVI1 and LAVI2. 
$(\mathrm{r}=0.88, \mathrm{p}<0.001)$ (Figure 1A). Bland Altman analysis showed a mean difference $( \pm \mathrm{SD}$ ) of $-0.75 \pm 8.8 \mathrm{~mL} / \mathrm{m} 2$ between $\mathrm{LAVI}_{1}$ and $\mathrm{LAVI}_{2}$, with limits of agreement $(-18.0,16.5)$ (Figure 1B).

\section{Conclusions}

LAVI determined by the Simpson's method of discs and the bi-plane area-length method correlate well in CMR imaging. Our data do not suggest the two methods can be used interchangeably given wide limits of agreement.

\section{Funding}

None.

Published: 16 January 2014

doi:10.1186/1532-429X-16-S1-P286

Cite this article as: Vongooru et al:: Quantification of left atrial volume using cardiac magnetic resonance imaging: comparison of left atrial volume index measurements using the Simpson's and bi-plane arealength methods. Journal of Cardiovascular Magnetic Resonance 201416 (Suppl 1):P286. 